\title{
A measurement of $\tau$ polarization at LEP
}

\section{L3 Collaboration}

M. Acciarri ${ }^{z}$, A. Adam ${ }^{\text {qq }}$, O. Adriani ${ }^{p}$, M. Aguilar-Benitez ${ }^{y}$, S. Ahlen ${ }^{j}, J_{.}$Alcaraz $^{q}$, A. Aloisio $^{\text {ab }}$, G. Alverson ${ }^{\text {k }}$, M.G. Alviggi ${ }^{\text {ab }}$, G. Ambrosi ${ }^{\text {ag }}$, Q. An ${ }^{\text {, }}$, H. Anderhub ${ }^{\text {at }}$, A.L. Anderson ${ }^{\circ}$, V.P. Andreev ${ }^{\text {ak }}$, T. Angelescu ${ }^{\ell}$, L. Antonov ${ }^{\text {an }}$, D. Antreasyan ${ }^{h}$, G. Alkhazov $^{\text {ak }}$, P. Arce ${ }^{y}$, A. Arefiev ${ }^{\text {aa }}$, T. Azemoon ${ }^{\text {c }}$, T. Aziz ${ }^{i}$, P.V.K.S. Baba ${ }^{r}$, P. Bagnaia ${ }^{\text {aj }}$, J.A. Bakken $^{\text {ai }}$, L. Baksay ${ }^{\text {ap }}$, R.C. Ball ${ }^{c}$, S. Banerjee ${ }^{i}$, K. Banicz ${ }^{\text {aq }}$, R. Barillère ${ }^{q}$, L. Barone $^{\text {aj }}$, A. Baschirotto ${ }^{z}$, M. Basile ${ }^{\text {h }}$, R. Battiston ${ }^{\text {ag }}$, A. Bay ${ }^{\text {s }}$, F. Becattini ${ }^{\mathrm{P}}$, U. Becker ${ }^{\circ}$, F. Behner ${ }^{\text {at }}$, Gy.L. Bencze $^{m}$, J. Berdugo ${ }^{y}$, P. Berges ${ }^{\circ}$, B. Bertucci ${ }^{\text {ag }}$, B.L. Betev ${ }^{\text {an }, a t}$, M. Biasini ${ }^{a g}$, A. Biland $^{\text {at }}$, G.M. Bilei ${ }^{\text {ag }}$, R. Bizzarri ${ }^{\text {aj }}$, J.J. Blaising ${ }^{\text {d }}$, G.J. Bobbink ${ }^{\text {q.b }}$, R. Bock ${ }^{\text {a }}$, A. Böhm ${ }^{a}$, B. Borgia ${ }^{\text {aj }}$, A. Boucham ${ }^{\text {d }}$, D. Bourilkov ${ }^{\text {at }}$, M. Bourquin ${ }^{\text {s }}$, D. Boutigny ${ }^{\text {q }}$, B. Bouwens ${ }^{b}$, E. Brambilla ${ }^{\circ}$, J.G. Branson ${ }^{a \ell}$, V. Brigljevic ${ }^{\text {at }}$, I.C. Brock ${ }^{\text {ah }}$, M. Brooks ${ }^{\text {w }}$, A. Bujak ${ }^{\text {aq }}$, J.D. Burger ${ }^{0}$, W.J. Burger ${ }^{\text {s }}$, C. Burgos ${ }^{y}$, J. Busenitz ${ }^{\text {ap }}$, A. Buytenhuijs ${ }^{\text {ad }}$, A. Bykov ${ }^{\text {ak }}$, X.D. Cai ${ }^{\text {r }}$, M. Capell ${ }^{\text {}}$, G. Cara Romeo ${ }^{\text {h }}$, M. Caria ${ }^{\mathrm{ag}}$, G. Carlino ${ }^{\mathrm{ab}}$, A.M. Cartacci ${ }^{\mathrm{p}}$, J. Casaus ${ }^{y}$, R. Castello ${ }^{z}$, N. Cavallo ${ }^{\text {ab }}$, M. Cerrada ${ }^{y}$, F. Cesaroni ${ }^{\text {aj }}$, M. Chamizo ${ }^{y}$, Y.H. Chang av , U.K. Chaturvedi ${ }^{r}$, M. Chemarin ${ }^{x}$, A. Chen ${ }^{\text {av }}$, C. Chen ${ }^{f}$, G. Chen ${ }^{\text {f,at }}$, G.M. Chen ${ }^{\mathrm{f}}$, H.F. Chen ${ }^{\mathrm{t}}$, H.S. Chen ${ }^{\mathrm{f}}$, M. Chen ${ }^{\circ}$, G. Chiefari ${ }^{\text {ab }}$, C.Y. Chien ${ }^{\mathrm{e}}$, M.T. Choi ${ }^{\text {ao }}$,

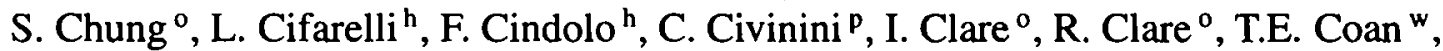
H.O. Cohn ${ }^{\text {ae }}$, G. Coignet ${ }^{d}$, N. Colino ${ }^{q}$, S. Costantini ${ }^{\text {aj }}$, F. Cotorobai ${ }^{\ell}$, B. de la Cruz ${ }^{y}$, X.T. Cui ${ }^{r}$, X.Y. Cui ${ }^{r}$, T.S. Dai ${ }^{\circ}$, R. D'Alessandro ${ }^{\text {P }}$, R. de Asmundis ${ }^{\text {ab }}$, A. Degré ${ }^{d}$, K. Deiters ${ }^{\text {ar }}$, E. Dénes ${ }^{m}$, P. Denes ${ }^{\text {ai }}$, F. DeNotaristefani ${ }^{\text {aj }}$, D. DiBitonto ${ }^{\text {ap }}$, M. Diemoz ${ }^{\text {aj }}$, H.R. Dimitrov ${ }^{\text {an }}$, C. Dionisi aj, M. Dittmar ${ }^{\text {at }}$, L. Djambazov ${ }^{\text {at }}$, I. Dorne ${ }^{\text {d }}$, M.T. Dova ${ }^{\text {r,3 }}$, E. Drago ${ }^{a b}$, D. Duchesneau ${ }^{\text {s }}$, F. Duhem ${ }^{d}$, P. Duinker ${ }^{b}$, I. Duran ${ }^{a m}$, S. Dutta ${ }^{i}$, S. Easo ${ }^{\text {ag }}$, H. El Mamouni ${ }^{x}$, A. Engler ${ }^{\text {ah }}$, F.J. Eppling ${ }^{\circ}$, F.C. Erné ${ }^{b}$, P. Extermann ${ }^{\text {s }}$, R. Fabbretti ${ }^{\text {ar }}$, M. Fabre ${ }^{\text {ar }}$, S. Falciano ${ }^{\text {aj }}$, A. Favara ${ }^{p}$, J. Fay $^{x}$, M. Felcini ${ }^{\text {at }}$, T. Ferguson ${ }^{\text {ah }}$, D. Fernandez ${ }^{y}$, G. Fernandez ${ }^{y}$, F. Ferroni ${ }^{\text {aj }}$, H. Fesefeldt ${ }^{a}$, E. Fiandrini ${ }^{\text {ag }}$, J.H. Field ${ }^{\text {s }}$, F. Filthaut ${ }^{\text {ad }}$, P.H. Fisher ${ }^{\mathrm{e}}$, G. Forconi ${ }^{\circ}$, L. Fredj ${ }^{\text {s }}$, K. Freudenreich ${ }^{\text {at }}$, M. Gailloud ${ }^{\text {v }}$, Yu. Galaktionov ${ }^{\text {aa,o }}$,

E. Gallo ${ }^{\text {p }, ~ S . N . ~ G a n g u l i ~}{ }^{\mathrm{i}}$, P. Garcia-Abia ${ }^{\mathrm{y}}$, S. Gentile ${ }^{\text {aj }}$, J. Gerald ${ }^{\mathrm{e}}$, N. Gheordanescu ${ }^{\ell}$, S. Giagu ${ }^{\text {aj }, ~ S . ~ G o l d f a r b ~}{ }^{\mathrm{v}}$, J. Goldstein ${ }^{\mathrm{j}}$, Z.F. Gong ${ }^{\mathrm{t}}$, E. Gonzalez ${ }^{\mathrm{y}}$, A. Gougas ${ }^{\mathrm{e}}$, D. Goujon ${ }^{\mathrm{s}}$, G. Gratta $^{\text {af }}$, M.W. Gruenewald ${ }^{\mathrm{g}}$, C. Gu ${ }^{\mathrm{r}}$, M. Guanziroli ${ }^{\mathrm{r}}$, V.K. Gupta ${ }^{\text {ai }}$, A. Gurtu ${ }^{\mathrm{i}}$, H.R. Gustafson ${ }^{c}$, L.J. Gutay ${ }^{\text {aq }}$, A. Hasan ${ }^{r}$, D. Hauschildt ${ }^{b}$, J.T. He ${ }^{f}$, T. Hebbeker $^{\mathrm{g}}$, M. Hebert ${ }^{a \ell}$, A. Hervé ${ }^{\mathrm{q}}$, K. Hilgers ${ }^{\mathrm{a}}$, H. Hofer ${ }^{\text {at }}$, H. Hoorani ${ }^{\text {s }}$, S.R. Hou ${ }^{\text {av }}$, G. Hu ${ }^{r}$, B. Ille ${ }^{\mathrm{x}}$, 
M.M. Ilyas ${ }^{\mathrm{r}}$, V. Innocente ${ }^{q}$, H. Janssen ${ }^{\text {d }}$, B.N. Jin ${ }^{\text {f }}$, L.W. Jones ${ }^{\text {c }}$, P. de Jong ${ }^{0}$, I. Josa-Mutuberria ${ }^{q}$, A. Kasser ${ }^{v}$, R.A. Khan ${ }^{r}$, Yu. Kamyshkov ${ }^{\text {ae }}$, P. Kapinos ${ }^{\text {as }}$, J.S. Kapustinsky ${ }^{\text {w }}$, Y. Karyotakis ${ }^{q}$, M. Kaur ${ }^{r}$, S. Khokhar ${ }^{\text {r }}$, M.N. Kienzle-Focacci ${ }^{\text {s }}$, D. Kim ${ }^{\mathrm{e}}$, J.K. Kim ${ }^{\text {ao }}$, S.C. Kim $^{\text {ao }}$, Y.G. Kim ${ }^{\text {ao }}$, W.W. Kinnison ${ }^{\text {w }}$, A. Kirkby ${ }^{\text {af }}$, D. Kirkby ${ }^{\text {af }}$, S. Kirsch ${ }^{\text {as }}$, W. Kittel ${ }^{\text {ad }}$, A. Klimentov ${ }^{\text {o,aa }}$, A.C. König ${ }^{\text {ad }}$, E. Koffeman ${ }^{\text {b }}$, O. Kornadt ${ }^{\text {a }}$, V. Koutsenko ${ }^{\text {o,aa }}$, A. Koulbardis ${ }^{\text {ak }}$, R.W. Kraemer ${ }^{\text {ah }}$, T. Kramer ${ }^{\circ}$, V.R. Krastev ${ }^{\text {an.ag, W. Krenz }}{ }^{\text {a }}$, H. Kuijten ${ }^{\text {ad, }}$, K.S. Kumar ${ }^{n}$, A. Kunin ${ }^{\text {o,aa }}$, P. Ladron de Guevara ${ }^{y}$, G. Landi ${ }^{\text {, }}$, D. Lanske ${ }^{a}$, S. Lanzano ${ }^{\text {ab, }}{ }^{\text {, }}$, A. Lebedev ${ }^{\circ}$, P. Lebrun ${ }^{x}$, P. Lecomte ${ }^{\text {at }}$, P. Lecoq ${ }^{9}$, P. Le Coultre ${ }^{\text {at }}$, D.M. Lee ${ }^{w}$, J.S. Lee $^{\text {ao }}$, K.Y. Lee ${ }^{\text {ao }}$, I. Leedom ${ }^{k}$, C. Leggett ${ }^{c}$, J.M. Le Goff ${ }^{q}$, R. Leiste ${ }^{\text {as }}$, M. Lenti ${ }^{p}$, E. Leonardi ${ }^{\text {aj }}$, P. Levtchenko ${ }^{\text {ak }}$, C. Li $^{\text {tr. }}$, E. Lieb ${ }^{\text {as }}$, W.T. Lin ${ }^{\text {av }}$, F.L. Linde ${ }^{b}$, B. Lindemann ${ }^{a}$, L. Lista ${ }^{\text {ab }}$, Y. Liu ${ }^{\text {r }}$, W. Lohmann ${ }^{\text {as }}$, E. Longo ${ }^{\text {aj, }}$ W. Lu ${ }^{\text {af }}$, Y.S. Lu ${ }^{\text {f }}$, J.M. Lubbers ${ }^{\mathrm{q}}$, K. Lübelsmeyer ${ }^{\text {a }}$, C. Luci $^{\text {aj }}$, D. Luckey $^{\circ}$, L. Ludovici ${ }^{\text {aj }}$, L. Luminari ${ }^{\text {aj }}$, W. Lustermann ${ }^{\text {ar }}$, W.G. Ma ${ }^{1}$, M. MacDermott ${ }^{\text {at }}$, M. Maity ${ }^{\mathrm{i}}$, L. Malgeri ${ }^{\text {aj }}$, R. Malik ${ }^{\mathrm{r}}$, A. Malinin ${ }^{\text {aa }}$, C. Maña ${ }^{\mathrm{y}}, \mathrm{S}$. Mangla ${ }^{\mathrm{i}}$, M. Maolinbay ${ }^{\text {at }}$, P. Marchesini at, A. Marin ${ }^{\mathrm{j}}$, J.P. Martin ${ }^{\mathrm{x}}$, F. Marzano ${ }^{\text {aj }}$, G.G.G. Massaro ${ }^{\mathrm{b}}$, K. Mazumdar ${ }^{\mathrm{i}}$, P. McBride ${ }^{\mathrm{n}}$, T. McMahon ${ }^{\text {aq }}$, D. McNally ${ }^{\text {al }}$, S. Mele ${ }^{\text {ab }}$, M. Merk $^{\text {ah }}$,

L. Merola ${ }^{\text {ab }}$, M. Meschini ${ }^{\text {p}}$, W.J. Metzger ${ }^{\text {ad }}$, Y. Mi $^{v}$, A. Mihul ${ }^{\ell}$, G.B. Mills ${ }^{w}$, Y. Mir ${ }^{r}$, G. Mirabelli aj, J. Mnich ${ }^{\text {a }}$, M. Möller ${ }^{\text {a }}$, V. Monaco ${ }^{\text {aj }}$, B. Monteleoni ${ }^{p}$, R. Morand ${ }^{d}$, S. Morganti ${ }^{\text {aj }}$, N.E. Moulai ${ }^{r}$, R. Mount ${ }^{\text {af }}$, S. Müller ${ }^{\text {a }}$, E. Nagy ${ }^{\mathrm{m}}$, M. Napolitano ${ }^{\text {ab }}$, F. Nessi-Tedaldi ${ }^{\text {at }}$, H. Newman ${ }^{\text {af }}$, M.A. Niaz ${ }^{r}$, A. Nippe ${ }^{\text {a }}$, H. Nowak ${ }^{\text {as }}$, G. Organtini ${ }^{\text {aj }}$, D. Pandoulas ${ }^{\text {a }}$, S. Paoletti ${ }^{\text {aj, }}$ P. Paolucci ${ }^{\text {ab }}$, G. Pascale ${ }^{\text {aj }}$, G. Passaleva ${ }^{\text {p.ag }}$, S. Patricelli ${ }^{\text {ab }}$, T. Paul ${ }^{\text {c}}$, M. Pauluzzi ${ }^{\text {ag }}$, C. Paus ${ }^{\text {a }}$, F. Pauss ${ }^{\text {at }}$, Y.J. Pei ${ }^{\text {a }}$, S. Pensotti ${ }^{2}$, D. Perret-Gallix ${ }^{d}$, A. Pevsner ${ }^{\mathrm{e}}$, D. Piccolo ${ }^{\text {ab }}$, M. Pieri ${ }^{\mathrm{q}}$, J.C. Pinto $^{\text {ah }}$, P.A. Piroué ${ }^{\text {ai }}$, E. Pistolesi ${ }^{\mathrm{P}}$, F. Plasil ${ }^{\text {ae }}$, V. Plyaskin ${ }^{\text {aa }}$, M. Pohl ${ }^{\text {at }}$, V. Pojidaev ${ }^{\text {aa,p }}$, H. Postema ${ }^{\circ}$, N. Produit ${ }^{\text {s }}$ J.M. Qian ${ }^{\text {c }}$, K.N. Qureshi ${ }^{\text {r }}$, R. Raghavan ${ }^{\text {, }}$, G. Rahal-Callot ${ }^{\text {at }}$, P.G. Rancoita ${ }^{\text {z }}$, M. Rattaggi ${ }^{\mathrm{z}}$, G. Raven ${ }^{\mathrm{b}}$, P. Razis ${ }^{\text {ac }}$, K. Read ${ }^{\text {ae }}$, M. Redaelli ${ }^{\text {}}$, D. Ren ${ }^{\text {at }}$, Z. Ren $^{\mathrm{r}}$, M. Rescigno ${ }^{\text {aj }}$, S. Reucroft ${ }^{\mathrm{k}}$, A. Ricker ${ }^{a}$, S. Riemann ${ }^{\text {as }}$, B.C. Riemers ${ }^{\text {aq }}$, K. Riles ${ }^{c}$, O. Rind ${ }^{c}$, H.A. Rizvi ${ }^{r}$, S. Ro ${ }^{\text {ao }}$, A. Robohm ${ }^{\text {at }}$, F.J. Rodriguez ${ }^{y}$, B.P. Roe ${ }^{c}$, M. Röhner ${ }^{a}$, S. Röhner ${ }^{a}$, L. Romero ${ }^{y}$, S. Rosier-Lees ${ }^{d}$, R. Rosmalen ${ }^{\text {ad }}$, Ph. Rosselet ${ }^{v}$, W. van Rossum ${ }^{b}$, S. Roth ${ }^{\text {a }}$, A. Rubbia ${ }^{\circ}$, J.A. Rubio ${ }^{q}$, H. Rykaczewski at, J. Salicio ${ }^{q}$, J.M. Salicio ${ }^{y}$, E. Sanchez ${ }^{y}$, G.S. Sanders ${ }^{\text {w }}$, A. Santocchia ${ }^{\text {ag }}$, M.E. Sarakinos ${ }^{\text {aq }, ~ S . ~ S a r k a r ~}{ }^{i}$, G. Sartorelli ${ }^{\text {r }}$, M. Sassowsky ${ }^{a}$, G. Sauvage ${ }^{d}$, C. Schäfer ${ }^{\text {a }}$, V. Schegelsky ak, D. Schmitz ${ }^{\text {a }}$, P. Schmitz ${ }^{\text {a }}$, M. Schneegans ${ }^{d}$, N. Scholz ${ }^{\text {at }}$, H. Schopper ${ }^{\text {au }}$, D.J. Schotanus ${ }^{\text {ad }}$, S. Shotkin ${ }^{\circ}$, H.J. Schreiber ${ }^{\text {as }}$, J. Shukla ${ }^{\text {ah }}$, R. Schulte ${ }^{a}$, K. Schultze ${ }^{a}$, J. Schwenke ${ }^{a}$, G. Schwering ${ }^{a}$, C. Sciacca ${ }^{\text {ab }}$, I. Scott ${ }^{n}$, R. Sehgal ${ }^{r}$, P.G. Seiler ${ }^{a r}$, J.C. Sens ${ }^{\text {q,b }}$, L. Servoli ${ }^{\text {ag }}$, I. Sheer ${ }^{a \ell}$, S. Shevchenko ${ }^{\text {af }}$, X.R. Shi ${ }^{\text {af }}$, E. Shumilov ${ }^{\text {aa }}$,

V. Shoutko ${ }^{\text {aa }}$, D. Son ${ }^{\text {ao }}$, A. Sopczak ${ }^{q}$, V. Soulimov ${ }^{\text {ab }}$, C. Spartiotis ${ }^{u}$, T. Spickermann ${ }^{\text {a }}$, P. Spillantini ${ }^{p}$, M. Steuer ${ }^{\circ}$, D.P. Stickland ${ }^{\text {ai }}$, F. Sticozzi ${ }^{\circ}$, H. Stone ${ }^{\text {ai }}$, K. Strauch ${ }^{n}$, K. Sudhakar ${ }^{\mathrm{i}}$, G. Sultanov ${ }^{\mathrm{r}}$, L.Z. Sun ${ }^{\text {t,r }}$, G.F. Susinno ${ }^{\text {s }}$, H. Suter ${ }^{\text {at }}$, J.D. Swain ${ }^{\mathrm{r}}$, A.A. Syed ${ }^{\text {ad }}$, X.W. Tang ${ }^{f}$, L. Taylor ${ }^{k}$, R. Timellini ${ }^{\text {h }}$, Samuel C.C. Ting ${ }^{\circ}$, S.M. Ting ${ }^{\circ}$, O. Toker ${ }^{\text {ag, }}$, M. Tonutti ${ }^{\text {a }}$, S.C. Tonwar ${ }^{\mathrm{i}}$, J. Tóth ${ }^{\mathrm{m}}, \mathrm{G}$. Trowitzsch ${ }^{\text {as }}$, A. Tsaregorodtsev ${ }^{\text {ak }}$, G. Tsipolitis ${ }^{\text {ah }}$, C. Tully ai, J. Ulbricht ${ }^{\text {at }}$, L. Urbán ${ }^{\mathrm{m}}$, U. Uwer ${ }^{\mathrm{a}}$, E. Valente ${ }^{\text {aj }}$, R.T. Van de Walle ${ }^{\text {ad }}$, 
I. Vetlitsky ${ }^{\text {aa }}$, G. Viertel ${ }^{\text {at }}$, P. Vikas ${ }^{r}, U$. Vikas $^{r}, M$. Vivargent $^{\mathrm{d}}$, H. Vogel ${ }^{\text {ah }}, H$. Vogt $^{\text {as }}$, I. Vorobiev ${ }^{\text {n,aa }}$, A.A. Vorobyov ${ }^{\text {ak }}$, An.A. Vorobyov ${ }^{\text {ak }}$, L. Vuilleumier ${ }^{\mathrm{v}}$, M. Wadhwa ${ }^{\mathrm{y}}$, W. Wallraff ${ }^{a}$, J.C. Wang ${ }^{\circ}$, X.L. Wang ${ }^{\mathrm{t}}$, Y.F. Wang ${ }^{\mathrm{o}}$, Z.M. Wang ${ }^{\mathrm{r}, \mathrm{t}}$, A. Weber ${ }^{\mathrm{a}}$, J. Weber ${ }^{\text {at }}$, R. Weill ${ }^{v}$, C. Willmott ${ }^{y}$, F. Wittgenstein ${ }^{\mathrm{q}}$, D. Wright ${ }^{\text {ai }}$, S.X. Wu ${ }^{\mathrm{r}}$, S. Wynhoff ${ }^{\mathrm{a}}$, Z.Z. Xu ${ }^{\mathrm{t}}$,

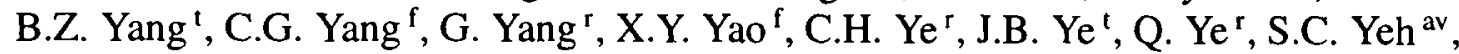
J.M. You ${ }^{\mathrm{r}}$, N. Yunus ${ }^{\mathrm{r}}$, M. Yzerman ${ }^{\mathrm{b}}$, C. Zaccardelli ${ }^{\text {af }}$, P. Zemp ${ }^{\mathrm{at}}$, M. Zeng ${ }^{\mathrm{r}}$, Y. Zeng ${ }^{\mathrm{a}}$, D.H. Zhang ${ }^{\text {b }}$, Z.P. Zhang ${ }^{\text {t,r }}$, B. Zhou ${ }^{j}$, G.J. Zhou ${ }^{f}$, J.F. Zhou ${ }^{a}$, R.Y. Zhu ${ }^{\text {af }}$, A. Zichichi ${ }^{\text {h,q,r }}$, B.C.C. van der Zwaan ${ }^{b}$

a I. Physikalisches Institut, RWTI, D-52056 Aachen, FRG ${ }^{1}$ III. Physikalisches Institut, RWTH, D-52056 Aachen, FRG ${ }^{1}$

b National Institute for High Energy Physics, NIKHEF, NL-1009 DB Amsterdam, The Netheriands

c University of Michigan, Ann Arbor, MI 48109, USA

'Laboratoire d'Annecy-le-Keux de Physique des Particules, LAPP,IN2P3-CNRS, BP 110, F-7494I Annecy-le-Keux CEIFX, France e Johns Hopkins University, Baltimore, MI) 21218, USA

f Institute of High Energy Physics, IHEP, 100039 Beijing, China g Humboldt Vniversity, D-10099 Berlin, FRG

h INFN-Secione di Bologna, I-40126 Bologna, Italy

i Tata Institute of Fundamental Rescarch, Bombay 400 005, India

i Boston University, Boston, MA 02215, USA

k Northeastem University, Boston, MA 02115, USA

'Institute of Atomic Physics and University of Bucharest, R-76900 Bucharest, Romania

$m$ Central Research Institute for Physics of the Hungarian Academy of Sciences, H-1525 Budapest 114, Hungary ${ }^{2}$

" Harvard University, Cambridge, MA 02139, USA

- Massachusetts Institute of Technology, Cambridge, MA 02199, USA

p INFN Sezione di Fircnze and University of Florence, I-50125 Florence, Italy

q Eurapean Laboratory for Particle Physics, CERN, CH-1211 Geneva 23, Surtzerland

r World Laboratory, FBLJA Projech, CH-1211 Gencva 23, Switzerland

- University of Geneva, CH-1211 Geneva 4, Switzerlond

${ }^{t}$ Chinese University of Science and Technology, USTC, Hefei, Anhui 230 029, China

" SEFT, Reseatch Institute for High Energy Physics, P.O. Box 9, SF-00014 Helsinki, Finland

$\checkmark$ University of Lausanne, CH-1015 Lausanne, Switzerland

w Los Alamos National Laboratory, Los Alamos, NM 87544, USA

× Institut de Physique Nucléaire de Iyon, IN2P3-CNRS,Université CTaude Bernard, F-69622 Villeurbanne Cedex, France

y Centro de Investigaciones tinergeticas, Medioambientalcs y Tecnologicas, CIEMAT, E-28040 Madrid, Spain

${ }^{2}$ INFN-Sczione di Milano, I-20133 Mrlan, Italy

as Institute of Theoretical and Expcrimental Physics, ITEP, Moscow, Russia

ab INFN-Sezione di Napoli and University of Naples, I-80125 Naples, Italy

a Department of Natural Sciences, University of Cyprus, Nicosia, Cyprus

ad University of Nymegen and NIKIIFF, NL-6525 ED Nymegen, The Netherlands

at Oak Ridge National Laboratory, Oak Ridge, TN 37831, USA

a California Institute of Technology, Pasadena, CA 91125, USA

as INFN-Sezione di Perugia and Universita Degli Studi di Perugia, I-06100 Perugia, Italy

ah Carnegie Mellon University, Pittsburgh, PA 15213, USA

ai Princeton University, Princeton, NJ 08544, USA

aj INFN-Sezione di Roma and University of Rome, "Ia Sapienza", I-00185 Rome, Italy

ak Nuclear Physics Institute, St. Petersburg, Russia

al University of Califormia, San Diego, CA 92093, USA

am Dept. de Fisica de Particulas Flementales, Univ. de Santiago, E-15706 Santiago de Compostela, Spain

an Bulgarion Academy of Sciences, Institute of Mechatronics, BU-1113 Sofia, Bulgaria

ao Center for High Fnergy Physics, Korca Advanced Inst. of Sciences and Tèchnology, 305-701 Tacjon, South Korea

ap University of Alabama, Tuscaloosa, AL 35486, USA

a Purdue University, West Lafayette, IN 47907, USA

ar Paul Scherrer Institut, PSI, CH-5232 Villigen, Suitzerland 
as DESY-Institut für Hochenergiephysik, D-15738 Zeuthen. FRG

at Eidgenössische Technische Hochschule, ETH Zürich, CH-8093 Zürich, Switzerland

au University of Hamburg, 22761 Hamburg, FRG

av High Energy Physics Group, Taiwan

Received 16 September 1994

Editor: K. Winter

\begin{abstract}
With a data sample of $86000 \mathrm{Z} \rightarrow \tau^{+} \tau^{-}(\gamma)$ events collected in 1990 through 1993 we have measured the polarization of $\tau$ leptons as a function of the production polar angle using the following 1-prong $\tau$ decay modes: $\tau^{-} \rightarrow \mathrm{e}^{-} \bar{\nu}_{\mathrm{e}} \nu_{\tau}, \tau^{-} \rightarrow \mu^{-} \bar{\nu}_{\mu} \nu_{\tau}$, $\tau^{-} \rightarrow \pi^{-}\left(\mathrm{K}^{-}\right) \nu_{\tau}, \tau^{-} \rightarrow \rho^{-} \nu_{\tau}$ and $\tau^{-} \rightarrow \mathrm{a}_{1}^{-} \nu_{\tau}$. We obtain for the ratio of vector to axial-vector weak neutral couplings for electrons $g \mathrm{Ve}_{\mathrm{ve}} / g_{\mathrm{Ae}}=0.0791 \pm 0.0099$ (stat) \pm 0.0025 (syst) and taus $g \mathrm{vv}_{\tau} / g_{\mathrm{Ar}}=0.0752 \pm 0.0063$ (stat) \pm 0.0045 (syst) consistent with the hypothesis of $\mathrm{e}-\tau$ universality. Assuming universality of the $\mathrm{e}-\tau$ neutral current we determine the effective electroweak mixing angle to be $\sin ^{2} \theta_{w}^{\text {eff }}=0.2309 \pm 0.0016$.
\end{abstract}

\section{Introduction}

In the reaction $\mathrm{e}^{+} \mathrm{e}^{-} \rightarrow \mathrm{Z} \rightarrow \tau^{+} \tau^{-}$even with unpolarized beams at $\sqrt{s} \approx M_{Z}$ the final state $\tau$ leptons are polarized. This polarization is due to the different couplings of left- and right-handed leptons to the $Z$ boson. The $\tau$ polarization, $\mathcal{P}_{\tau}(\cos \theta)$, is defined as the asymmetry in the production cross section of $\tau^{-}$leptons with positive helicity $(h=+1 / 2)$ and negative helicity $(h=-1 / 2)$

$\mathcal{P}_{\tau^{-}}(\cos \theta) \equiv \frac{\sigma(h=+1 / 2)-\sigma(h=-1 / 2)}{\sigma(h=+1 / 2)+\sigma(h=-1 / 2)}$,

where $\theta$ is the angle between the $\mathrm{e}^{-}$beam and $\tau^{-}$flight direction. The $\tau^{-}$and $\tau^{+}$leptons in each event have opposite helicity, so that $\mathcal{P}_{\tau^{-}}(\cos \theta)=$ $-\mathcal{P}_{\tau^{+}}(\cos \theta) \equiv \mathcal{P}_{\tau}(\cos \theta)$. In the improved Born approximation $\mathcal{P}_{\tau}(\cos \theta)$ at the $\mathrm{Z}$ pole is given by [1]:

$\mathcal{P}_{\tau}(\cos \theta)=-\frac{\mathcal{A}_{\tau}+2 \mathcal{A}_{e} \cos \theta /\left(1+\cos ^{2} \theta\right)}{1+2 \mathcal{A}_{\tau} \mathcal{A}_{\mathrm{e}} \cos \theta /\left(1+\cos ^{2} \theta\right)}$.

\footnotetext{
'Supported by the German Bundesministerium für Forschung und Technologie.

2 Supported by the Hungarian OTKA fund under contract number 2970.

${ }^{3}$ Also supported by CONICET and Universidad Nacional de La Plata, CC 67, 1900 La Plata, Argentina.

${ }^{4}$ Deceased.
}

The quantities $\mathcal{A}_{\ell}(\ell=\mathrm{e}, \tau)$ are defined as $\mathcal{A}_{\ell} \equiv$ $2 g_{\mathrm{V} \ell} g_{\mathrm{A} \ell} /\left(g_{\mathrm{V} \ell}^{2}+g_{\mathrm{A} \ell}^{2}\right)$, where $g_{\mathrm{V} \ell}$ and $g_{\mathrm{A} \ell}$ denote the effective vector and axial-vector coupling constants.

$\mathcal{P}_{\tau}$, the average of $\mathcal{P}_{\tau}(\cos \theta)$ over all production angles, is equivalent to $-\mathcal{A}_{r}$, which is independent of the coupling constants of the initial state electrons. The measurement of $\mathcal{P}_{\tau}(\cos \theta)$ yields both $\mathcal{A}_{\tau}$ and $\mathcal{A}_{\mathrm{e}}$, thus making it possible to check whether the $\mathrm{e}$ and $\tau$ couplings to the $\mathrm{Z}$ are equal, as required by the lepton universality hypothesis. In the framework of the Standard Model [2] the lepton couplings are equal and we use the average of $\mathcal{A}_{r}$ and $\mathcal{A}_{e}$ to determine the effective electroweak mixing angle through $g_{\mathrm{V} \ell} / g_{\mathrm{A} \ell}=$ $1-4 \sin ^{2} \theta_{w}^{\text {eff }}$.

In this analysis it is assumed that the decays of the $\tau$ are described by a pure $\mathrm{V}-\mathrm{A}$ weak charged current as supported by other measurements [3-5]. The polarization can be derived either from the analysis of the kinematics of single $\tau$ decay products [ 1,6-8] (single $\tau$ method) or from the acolinearity between the decay products of the $\tau$ pairs [9]. The following 1-prong $\tau$ decay channels have been used in this analysis:

$$
\begin{aligned}
& \tau^{-} \rightarrow \mathrm{e}^{-} \bar{\nu}_{\mathrm{e}} \nu_{\tau}, \quad \tau^{-} \rightarrow \mu^{-} \bar{\nu}_{\mu} \nu_{\tau}, \\
& \tau^{-} \rightarrow \pi^{-}\left(\mathrm{K}^{-}\right) \nu_{\tau}, \\
& \tau^{-} \rightarrow \rho^{-} \nu_{\tau}, \quad \tau^{-} \rightarrow \mathrm{a}_{1}^{-} \nu_{\tau}
\end{aligned}
$$

where the charge conjugate decays are implied here and throughout this paper. Pions and kaons are not distinguished in the hadronic decay channels. 
The analysis of the angular dependence of $\tau$ polarization described in this paper improves upon our earlier analysis [10] which was confined to the measurement of the average $\tau$ polarization. In addition we implement better techniques for particle identification, extend the geometrical acceptance from $|\cos \theta|<0.7$ to the forward-backward region (up to $|\cos \theta|=0.94$ ) and use a much larger data sample. The results presented below, based on a data sample of $86000 \tau$ pairs from a total integrated luminosity of $74 \mathrm{pb}^{-1}$ collected in the center of mass energy range $88.2<$ $\sqrt{s}<94.3 \mathrm{GeV}$ in the $1990-1993$ running periods, supersede (and are compatible with) our earlier results [10].

\section{Selection of $\tau$ decays}

The $\mathbf{L} 3$ detector is described in detail in Ref. [11]. The $\mathrm{e}^{+} \mathrm{e}^{-}$collision point is surrounded by a tracking chamber, a high resolution electromagnetic calorimeter, a cylindrical shell of scintillation counters, a hadron calorimeter and a muon chamber system. The detector is installed in a large magnet providing a uniform 0.5 Tesla field along the beam direction.

The selection of $\tau$ decays procecds in three steps. First, a sample of low multiplicity, back-to-back events is preselected, which consists mainly of leptons from Z decays. This preselection suppresses such background as cosmic muons, hadronic $\mathrm{Z}$ decays, two-photon and beam-gas interactions. In the second step the individual $\tau$ decays are identified. The identification is done independently in two hemispheres separated by a plane perpendicular to the thrust axis of the event. Particle identification in each hemisphere is based upon the topological properties of the energy deposition in the electromagnetic and hadron calorimeters with respect to the trajectory of the charged track. This technique is found to be relatively independent of the energy of the $\tau$-decay products, so polarization bias is minimal. Finally requirements are placed on the hemisphere opposite to the $\tau$ decay candidate in order to suppress the non- $\tau$ background. The final data sample consists of events in which at least one of the $\tau$ 's decays into one of the 5 channels listed in the introduction. Selection efficiencies are calculated using Monte Carlo simulation of $\mathrm{Z} \rightarrow \tau^{+} \tau^{-}(\gamma)$ [12] including full simulation

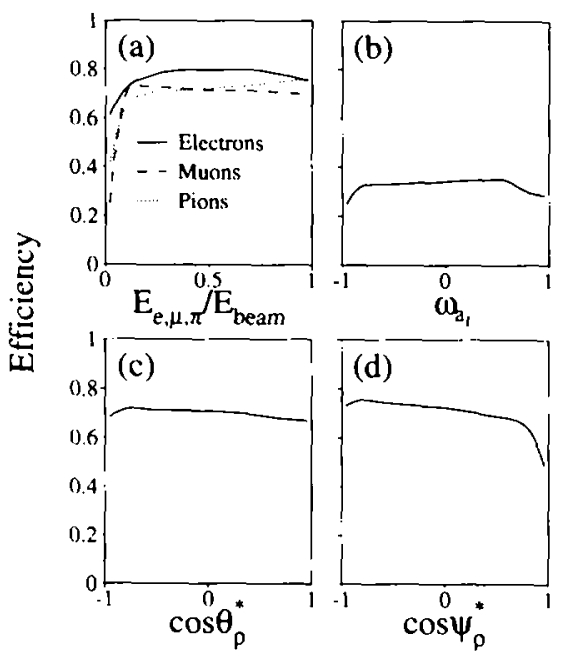

Fig. 1. All efficiencies in this figure correspond to the central region of the detector $|\cos \theta|<0.70$. (a) Selection efficiency for $\tau^{-} \rightarrow \mathrm{e}^{-} \bar{\nu}_{\mathrm{c}} \nu_{\tau}$ (solid line), $\tau^{-} \rightarrow \mu^{-} \bar{\nu}_{\mu} \nu_{\tau}$ (dashed line) and $\tau^{-} \rightarrow \pi^{-}\left(\mathrm{K}^{-}\right) \nu_{\tau}$ (dotted line) decays as a function of $E_{\pi, \mu, \pi} / E_{\text {beam }}$. (b) Selection cfficiency for $\tau^{-} \rightarrow \mathrm{a}_{1}^{-} \nu_{\tau}$ decays as a function of the polarization sensitive variable $\omega_{\mathrm{a}_{1}}$. (c) Selection efficiency for $\tau^{-} \rightarrow \rho^{-} \nu_{\tau}$ decays as a function of $\cos \theta_{\rho}^{*} \cdot \cos \theta_{\rho}^{*}$ is proportional to the sum of $\pi^{-}$and $\pi^{0}$ energies. (d) Selection efficiency for $\tau^{-} \rightarrow \rho^{-} \nu_{\tau}$ decays as a function of $\cos \psi_{\rho}^{*} \cdot \cos \psi_{\rho}^{*}$ is proportional to the difference of $\pi^{-}$and $\pi^{0}$ energies.

of the L3 detector response ${ }^{5}$. The background contamination from non- $\tau$ sources such as $\mathrm{Z} \rightarrow \mathrm{e}^{+} \mathrm{e}^{-}(\gamma)$, $\mathrm{Z} \rightarrow \mu^{+} \mu^{-}(\gamma), \mathrm{Z} \rightarrow$ hadrons, two-photon reactions, cosmics and beam-gas interactions is estimated primarily from the data using either the side opposite to the selected decay or global event characteristics. The corresponding Monte Carlo for the background simulation [15] is used only to cross-check these estimates.

Selection of $\tau^{-} \rightarrow \mathrm{e}^{-} \bar{\nu}_{\mathrm{e}} \nu_{\tau}$ and $\tau^{-} \rightarrow \mu^{-} \bar{\nu}_{\mu} \nu_{\tau}$ decays is similar to that described in Ref. [10]. The selection efficiency for $\tau^{-} \rightarrow \mathrm{e}^{-} \bar{\nu}_{\mathrm{e}} \nu_{\tau}$ is estimated to be $76 \%$ inside the fiducial region $|\cos \theta|<0.7$ and is independent of electron energy above $8 \mathrm{GeV}$ (Fig. la). The backgrounds are $1.5 \%$ from other $\tau$ decays, $1.6 \%$ from $\mathrm{Z} \rightarrow \mathrm{e}^{+} \mathrm{e}^{-}(\gamma)$ and 1.2\% from two-photon interactions. The selection efficiency for $\tau^{-} \rightarrow \mu^{-} \bar{\nu}_{\mu} \nu_{\tau}$ is $70 \%$ inside the fiducial region $|\cos \theta|<0.8$ and is independent of the muon momentum above $4 \mathrm{GeV}$

\footnotetext{
${ }^{5}$ The $\mathrm{L} 3$ detector simulation is based on GEANT Version 3.14; see Ref. [13]. The GHEISHA program [14] is used to simulate hadronic interactions.
} 
(Fig. 1a). The background contributions are $1.0 \%$ from other $\tau$ decays, $3.1 \%$ from $Z \rightarrow \mu^{+} \mu^{-}(\gamma), 0.8 \%$ from two-photon reactions and $0.3 \%$ from cosmics.

For the selection of the hadronic $\tau$ decays the hemispheres with identified electron or muon candidates are first rejected. Then an algorithm [10] for finding overlapping neutral energy clusters in the vicinity of hadronic shower in the electromagnetic calorimeter is applied in order to determine the number of neutral clusters and their energies. The invariant mass of each neutral cluster is estimated by fitting its transverse profile with the sum of two electromagnetic shower shapes. A single neutral cluster forms a $\pi^{0}$ candidate if its energy exceeds $1 \mathrm{GeV}$ and its transverse energy profile is consistent with an electromagnetic profile or its invariant mass is within $50 \mathrm{MeV}$ of the $\pi^{0}$ mass. Two distinct neutral clusters form a $\pi^{0}$ candidate if their invariant mass is within $40 \mathrm{MeV}$ of the $\pi^{0}$ mass. The sum of energies of reconstructed neutral clusters is subtracted from the energy in the electromagnetic calorimeter and the remaining energy along with the energy deposition in the hadron calorimeter is assigned to the charged hadron. This measurement of the calorimetric energy of the charged hadron is then combined with the independent measurement of the momentum in the tracking chamber by maximizing the likelihood for these two measurements to originate from a single hadron.

The $\tau^{-} \rightarrow \pi^{-}\left(\mathrm{K}^{-}\right) \nu_{\tau}$ selection admits no $\pi^{0}$ candidates and no neutral clusters with energy greater than $0.5 \mathrm{GeV}$. The probability that the observed track momentum and calorimetric energy originate from a single hadron is required to exceed 0.003 . In order to reject background from $\mathrm{Z} \rightarrow \mathrm{e}^{+} \mathrm{e}^{-}(\gamma)$ and $\mathrm{Z} \rightarrow$ $\mu^{+} \mu^{-}(\gamma)$, events with an electron or a muon candidate on the opposite side are rejected if its energy excceds $42 \mathrm{GeV}$ or can not be reliably measured. The efficiency of $\tau^{-} \rightarrow \pi^{-}\left(\mathrm{K}^{-}\right) \nu_{\tau}$ selection is $72 \%$ in the barrel $(|\cos \theta|<0.7)$ and $64 \%$ in the endcap $(0.82<$ $|\cos \theta|<0.94)$ region. The efficiency is relatively independent of the pion energy above $5 \mathrm{GeV}$ (Fig. la). The background in the barrel is $11.4 \%$ from other $\tau$ decays, $1.4 \%$ from Bhabha events, $1.4 \%$ from twophoton interactions and $0.9 \%$ from dimuon events; the corresponding numbers for the endcaps are $16 \%, 10 \%$, $1.5 \%$ and $5 \%$.

To select a $\tau^{-} \rightarrow \mathrm{a}_{1}^{-} \nu_{\tau}$ decay two $\pi^{0}$ candidates are required in the hemisphere. If the two $\pi^{0}$ candidates each consist of a single neutral cluster, then the invariant mass of these two neutral clusters must be incompatible with the mass of a $\pi^{0}$. The probability that the measured track momentum and calorimetric energy originate from a single hadron is required to exceed 0.001 . The selected decays are next subjected to a neural network selection in order to further reduce background from $\tau^{-} \rightarrow \rho^{-} \nu_{\tau}$ and $\tau^{-} \rightarrow \pi^{-} \pi^{0} \pi^{0} \pi^{0} \nu_{\tau}$ events. This neural network uses as an input ten variables corresponding to the energies of the charged pion and identified neutral clusters and the invariant masses of their combinations. The final selection efficiency is $33 \%$ in the fiducial volume $\left|\cos \theta_{\text {thrust }}\right|<0.7$. Fig. $1 \mathrm{~b}$ shows the selection efficiency as a function of the polarization sensitive variable $\omega_{\mathrm{a}_{1}}$ [16]. This variable is a combination of three decay angles (the decay angle of the $a_{1}^{-}$in the $\tau^{-}$rest frame, the decay angle of three pions in the $a_{1}^{-}$rest frame and the orientation angle of $\pi^{-} \pi^{0} \pi^{0}$ system in the $\mathrm{a}_{1}^{-}$decay plane) and three invariant masses $\left(\pi^{-} \pi^{0} \pi^{0}\right.$ mass and two $\pi^{-} \pi^{0}$ masses). In order to reject the background from $\mathrm{Z} \rightarrow \mathrm{e}^{+} \mathrm{e}^{-}(\gamma)$, events with an electron candidate in the opposite hemisphere are rejected if its energy exceeds $40 \mathrm{GeV}$ or cannot be reliably measured. This reduces background from the non- $\tau$ sources to a negligible level. The background from other $\tau$ decays is $28 \%$.

To select a $\tau^{-} \rightarrow \rho^{-} \nu_{\tau}$ decay exactly one $\pi^{0}$ candidate is required in the hemisphere. The invariant mass of $\pi^{-} \pi^{0}$ system must be in the range $0.45-1.20$ $\mathrm{GeV}$. The probability that the measured track momentum and calorimetric energy originate from a single hadron is required to exceed 0.001 . In order to reject background from $Z \rightarrow \mathrm{e}^{+} \mathrm{e}^{-}(\gamma)$ and $\mathrm{Z} \rightarrow \mu^{+} \mu^{-}(\gamma)$, events with an electron or a muon candidate in the opposite hemisphere are rejected if its energy exceeds 42 $\mathrm{GeV}$ or cannot be reliably measured. The efficiency of the selection is $70 \%$ in the barrel and $51 \%$ in the endcap. Figs. 1c,d show the selection efficiency as a function of the two polarization sensitive variables $\theta_{\rho}^{*}$, the decay angle of the $\rho^{-}$in the $\tau^{-}$rest frame, and $\psi_{\rho}^{*}$, the decay angle of the $\pi^{-}$in the $\rho^{-}$rest frame. The background in the barrel is $10.2 \%$ from other $\tau$ decays, $0.2 \%$ from $\mathrm{Z} \rightarrow \mathrm{e}^{+} \mathrm{e}^{-}(\gamma)$ and $0.5 \%$ from $Z \rightarrow \mu^{+} \mu^{-}(\gamma)$. In the endcap the numbers are $14.3 \%$, $1.5 \%$ and $1.5 \%$, respectively. Fig. $2 \mathrm{a}, \mathrm{b}$ show the invariant mass spectra of $\pi^{0}$ and $\pi^{-} \pi^{0}$, respectively, for the selected sample of $\tau^{-} \rightarrow \rho^{-} \nu_{\tau}$ events. 

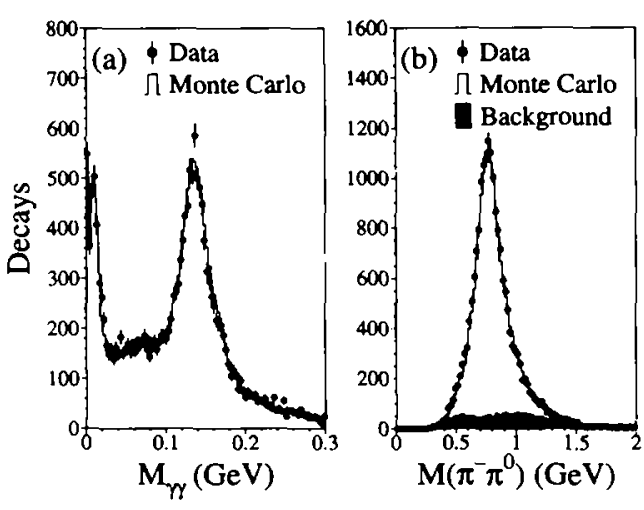

Fig. 2. (a) Mass of $\gamma \gamma$ pairs for selected $\tau^{-} \rightarrow \rho^{-} \nu_{\tau}$ candidates showing a clear $\pi^{0}$ peak. (b) Mass spectrum of $\pi^{-} \pi^{0}$ of $\tau^{-} \rightarrow \rho^{-} \nu_{\tau}$ candidates in the range $0<M_{\pi^{-} \pi^{0}}<2 \mathrm{GeV}$.

\section{Measurement of the polarization}

The goal of this measurement is the determination of the ratios of vector to axial-vector weak neutral couplings for electrons and taus. The analysis proceeds in three steps. First the angular dependence of $\mathcal{P}_{\tau}$ is obtained by measuring the polarization in nine regions of polar angle. The sizes of the $\cos \theta$ regions are chosen such that they all contain approximately the same number of events. The measurement of the polarization in each $\cos \theta$ region is performed separately for every decay channel using the single $\tau$ method, and is also performed using the acolinearity method. Next $\mathcal{A}_{e}$ and $\mathcal{A}_{r}$ are determined for each decay channel from the corresponding $\mathcal{P}_{\tau}(\cos \theta)$ dependence. Finally all the individual $\mathcal{P}_{\tau}$ measurements are combined bin-bybin and final values of $\mathcal{A}_{e}$ and $\mathcal{A}_{\tau}$ are determined.

\subsection{Charge identification}

The polar angle of the selected $\tau$ candidate is defined by $\left|\cos \theta_{\text {thrust }}\right|$ assigned according to the event charge, defined as the charge of the $\tau$ traveling into the forward $(\cos \theta>0)$ hemisphere. For events with at least one $\tau^{-} \rightarrow \mu^{-} \bar{\nu}_{\mu} \nu_{\tau}$ decay, the event charge is unambiguously assigned by the muon chambers. For events with no identified muons but exactly one track in each hemisphere the charge is defined by the sign of the difference of curvatures weighted by resolutions for the two tracks. For all other events the charge is not defined and these are used for the measurement of average polarization only.
The sign of the polar angle can be misassigned due to charge confusion, causing events to migrate between $\cos \theta$ bins of opposite sign. This migration changes the measured polarization in each $\cos \theta$ bin, and thus affects the shape of the $\mathcal{P}_{\tau}(\cos \theta)$ distribution. The measured resolution of the tracking chamber is used to determine the charge confusion as a function of the momentum and polar angle of the charged $\tau$ decay product, and a correction for its effect on polarization is applied in each $\cos \theta$ bin. The average charge confusion at $P=45 \mathrm{GeV}$ is found to be about $3 \%$ in the barrel and $7 \%$ in the endcap. The corresponding numbers for an average $\mathrm{Z} \rightarrow \tau^{+} \tau^{-}$event are $1.2 \%$ and $3 \%$, respectively.

\section{2. $\mathcal{P}_{\tau}$ fitting procedure}

For each $\tau$ decay channel, $\mathcal{P}_{\tau}$ is measured by obtaining the linear combination of the $h=+1 / 2, h=$ $-1 / 2$ Monte Carlo and the non- $\tau$ background distributions which best fits the data. We use a binned maximum likelihood function which properly accounts for the finite statistics both in the data and in the Monte Carlo. This is verified by using the likelihood function to fit samples created by a fast detector simulation which generates the distributions of polarization sensitive variables for each $\tau$ decay channel and includes the effects of acceptance and background contamination. Samples with statistics comparable to that in the data and in the full detector Monte Carlo are fitted. The fit errors are then compared to the actual spread of fitted values, and are found to agree.

The polarization of the background from other $\tau$ decays is varied simultaneously with the polarization for the decay mode being fitted. The normalizations of the backgrounds from $\mathrm{Z} \rightarrow \mathrm{e}^{+} \mathrm{e}^{-}(\gamma)$ and $\mathrm{Z} \rightarrow \mu^{+} \mu^{-}(\gamma)$ are left as free parameters in the fits for the $\tau^{-} \rightarrow$

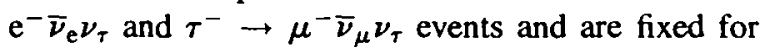
$\tau^{-} \rightarrow \pi^{-}\left(\mathrm{K}^{-}\right) \nu_{\tau}, \tau^{-} \rightarrow \rho^{-} \nu_{\tau}$ and $\tau^{-} \rightarrow \mathrm{a}_{1}^{-} \nu_{\tau}$ decays. The contribution of all other background sources (mainly from two-photon reactions and cosmics) is estimated from the data itself and is fixed in the fit for all the channels. The energy spectra of the selected electrons, muons and pions used for the polarization measurement are shown in Figs. 3a-c, together with the best fit Monte Carlo distributions. For the energy spectrum of pions we use non-equidistant binning in order to reduce the resolution effects at high energy. 

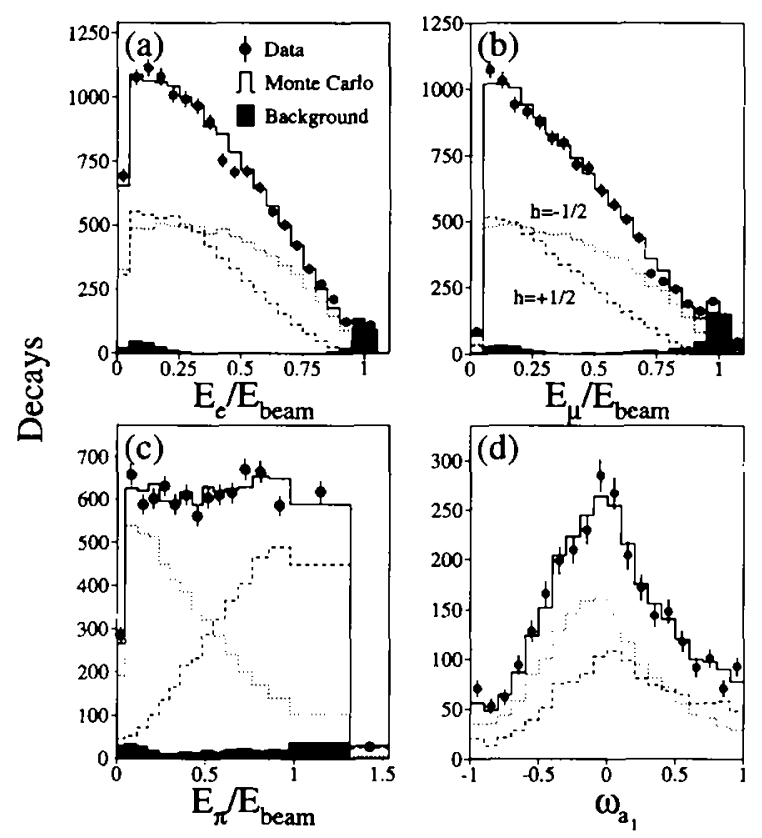

Fig. 3. (a) Spectrum for $\tau^{-} \rightarrow \mathrm{e}^{-} \bar{\nu}_{\mathrm{e}} \nu_{\tau}$ decays as a function of $E_{\mathrm{c}} / E_{\text {beam }}$ showing the Monte Carlo best fit, the contribution from each helicity, and non- $\tau$ background. (b) Spectrum for $\tau^{-} \rightarrow \mu^{-} \bar{\nu}_{\mu} \nu_{\tau}$ decays as a function of $E_{\mu} / E_{\text {beam }}$. (c) Spectrum for $\tau^{-} \rightarrow \pi^{-}\left(\mathrm{K}^{-}\right) \nu_{\tau}$ decays as a function of $E_{\pi} / E_{\text {beam }}$. Nonequidistant energy binning is used to reduce the effect of resolution at high energies. (d) Spectrum for $\tau^{-} \rightarrow a_{1}^{-} \nu_{\tau}$ decays as a function of $\omega_{\mathrm{a}_{1}}$.

To fit $\tau^{-} \rightarrow \mathrm{a}_{1}^{-} \nu_{\tau}$ decays we use the optimal variable fitting [17] following the strategy of Ref. [16] for the construction of the polarization sensitive variable $\omega_{\mathrm{a}_{1}}$. A study of the hadronic structure functions of the $a_{1}$ has been performed in order to discriminate between various theoretical models [18-20]. Our data is in qualitative agreement with the model described in Ref. [18], which is therefore used in the analysis. The $\omega_{\mathrm{a}_{1}}$ spectrum for the selected events is shown in Fig. 3d, together with the best fit Monte Carlo distributions.

For the analysis of the decay mode $\tau^{-} \rightarrow \rho^{-} \nu_{\tau}$ we fit a $10 \times 15$ matrix in the parameter space of $\cos \theta_{\rho}^{*}$ and $\cos \psi_{\rho}^{*}$. The spectra of $\cos \psi_{\rho}^{*}$ in four slices of $\cos \theta_{\rho}^{*}$ for the selected decays are shown in Fig. 4, together with the best fit Monte Carlo distributions. We also implement the optimal variable fitting and find the results of two approaches to be in a good agreement. The quoted numbers refer to the two-dimensional fit which exhibits slightly better sensitivity due to the

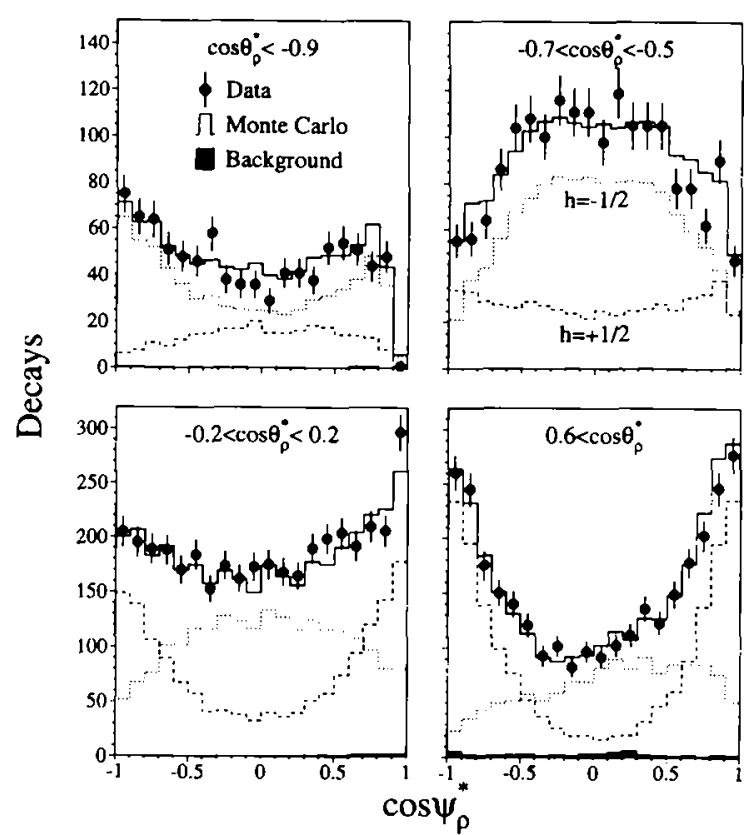

Fig. 4. The spectra for $\tau^{-} \rightarrow \rho^{-} \nu_{\tau}$ as a function of $\cos \psi_{\rho}^{*}$ in four ranges of $\cos \theta_{\rho}^{*}$. Also shown are the Monte Carlo best fit, the contribution from each helicity, and the non- $\tau$ background. The ranges of $\cos \theta_{\rho}^{*}$ are chosen to bring out the features of $\cos \psi_{\rho}^{*}$ distributions which provide the distinguishing power for decays of $\tau^{-}$leptons with positive and negative helicity states.

better separation of the signal and non- $\tau$ background.

Using $\tau^{-} \rightarrow \rho^{-} \nu_{\tau}$ decays we have measured $\mathcal{P}_{\tau}$ as a function of $M_{\pi-\pi^{0}}$. An indication for a possible dependence of $\mathcal{P}_{\tau}$ on $M_{\pi^{-} \pi^{0}}$ was observed in the 19911992 data sample. The dependence is not seen with the 1993 data sample which is of comparable statistical significance. A number of checks were performed assuring the stability of the detector during the 19911993 running periods. We conclude that the fluctuation seen in the 1991-1992 data sample is of a pure statistical nature.

\subsection{Systematic uncertainties in $\mathcal{P}_{\tau}$}

Systematic errors in the measurement of the polarization arise from the selection procedure, background estimation, calibration, charge confusion and theoretical uncertainties. The corresponding uncertainty in the polarization is estimated by varying the most important selection cuts, the background contamination, the energy scales of different subdetectors, or by chang- 
Table 1

Summary of the systematic uncertainties in $\mathcal{P}_{\tau}$. The estimation is listed for the $\cos \theta$ range $[-0.72,-0.55]$.

\begin{tabular}{|c|c|c|c|c|c|c|c|}
\hline \multicolumn{2}{|l|}{ Error due to } & \multirow{2}{*}{$\frac{\mathrm{e}^{-\bar{\nu}_{\mathrm{e}} \nu_{\tau}}}{0.010}$} & \multirow{2}{*}{$\frac{\mu-\bar{\nu}_{\mu} \nu_{\tau}}{0.006}$} & \multirow{2}{*}{$\frac{\pi^{-}\left(\mathrm{K}^{-}\right) \nu_{\tau}}{0.007}$} & \multirow{2}{*}{$\frac{\rho^{-} \nu_{T}}{0.008}$} & \multirow{2}{*}{$\frac{\mathrm{a}_{1}^{-} \nu_{\tau}}{0.020}$} & \multirow{2}{*}{$\frac{\text { acolinearity }}{0.010}$} \\
\hline selection & $\Delta \mathcal{P}_{\mathcal{S}}^{\text {sel }}$ & & & & & & \\
\hline $\begin{array}{l}\text { background } \\
\text { calibration }\end{array}$ & $\begin{array}{l}\Delta \mathcal{P}_{\tau}^{\text {hq }} \\
\Delta \mathcal{P}_{\tau}^{\text {cal }}\end{array}$ & $\begin{array}{l}0.017 \\
0.014\end{array}$ & $\begin{array}{l}0.022 \\
0.016\end{array}$ & $\begin{array}{l}0.008 \\
0.026\end{array}$ & $\begin{array}{l}0.004 \\
0.014\end{array}$ & $\begin{array}{l}0.010 \\
0.020\end{array}$ & $\begin{array}{l}0.010 \\
0.016\end{array}$ \\
\hline $\begin{array}{l}\text { charge confusion } \\
\text { theory }\end{array}$ & $\begin{array}{l}\Delta \mathcal{P}_{\tau}^{\text {chrg }} \\
\Delta \mathcal{P}_{\tau}^{\text {theor }}\end{array}$ & $\begin{array}{r}0.002 \\
<0.001\end{array}$ & $\begin{array}{l}<0.001 \\
<0.001\end{array}$ & $\begin{array}{l}0.003 \\
0.002\end{array}$ & $\begin{array}{r}0.002 \\
<0.001\end{array}$ & $\begin{array}{l}0.002 \\
0.015\end{array}$ & $\begin{array}{l}0.003 \\
0.002\end{array}$ \\
\hline
\end{tabular}

ing the underlying theoretical assumptions. The corresponding change in the fitted value of $\mathcal{P}_{\tau}$ represents the systematic error.

The systematic error due to uncertainty in the rate of incorrectly identified $\tau$ decays is estimated by varying the corresponding branching fractions according to the uncertainties of our measurements for $\tau^{-} \rightarrow \mathrm{e}^{-} \bar{\nu}_{\mathrm{e}} \nu_{\tau}$, $\tau^{-} \rightarrow \mu^{-} \bar{\nu}_{\mu} \nu_{\tau}, \tau^{-} \rightarrow \pi^{-}\left(\mathrm{K}^{-}\right) \nu_{\tau}, \tau^{-} \rightarrow \rho^{-} \nu_{\tau}$ and $\tau^{-} \rightarrow \mathrm{a}_{1}^{-} \nu_{\tau}[21,22]$ and uncertaintics in PDG valucs [23] for $\tau$ decays involving kaons. Background from $\mathrm{Z} \rightarrow \mathrm{c}^{+} \mathrm{e}^{-}(\gamma), \mathrm{Z} \rightarrow \mu^{+} \mu^{-}(\gamma)$, two-photon interactions and cosmics is estimated independently for each $\cos \theta$ bin, and each of these has a typical statistical uncertainty in the normalization of $10-20 \%$. The systematic error due to the uncertainty in the shape of the non- $\tau$ background is negligible compared with that from the normalization.

The accuracy of the energy scale for electrons and photons is estimated to be $1 \%$ at $1 \mathrm{GeV}$ from the measurement of the position of the $\pi^{0}$ peak and $0.1 \%$ at $45 \mathrm{GeV}$ from the study of $\mathrm{Z} \rightarrow \mathrm{e}^{+} \mathrm{c}^{-}(\gamma)$ events. The effect of this uncertainty on the $\tau^{-} \rightarrow \rho^{-} \nu_{\tau}$ channel is opposite to the effect on $\tau^{-} \rightarrow \mathrm{e}^{-} \bar{\nu}_{\mathrm{e}} \nu_{\tau}$ channel. The momentum scale of the tracking chamber is verified to $1 \%$ accuracy from 1 to $45 \mathrm{GeV}$ using low energy electrons as well as muons from $\tau$ and $\mathrm{Z}$ decays. This uncertainty affects the polarization measurement in the $\tau^{-} \rightarrow \pi^{-}\left(\mathrm{K}^{-}\right) \nu_{\tau}, \tau^{-} \rightarrow \rho^{-} \nu_{\tau}$ and $\tau^{-} \rightarrow \mathrm{a}_{1}^{-} \nu_{\tau}$ channels in the same way. The accuracy of the muon momentum scale is estimated to be $0.2 \%$ at $45 \mathrm{GeV}$ using $\mathrm{Z} \rightarrow \mu^{+} \mu^{-}(\gamma)$ events. At low momenta, the uncertainty in the muon momentum scale is dominated by the muon energy loss in the calorimeters which is known to an accuracy of $50 \mathrm{MeV}$. The absolute energy scales of the electromagnetic and hadron calorimeters for hadrons are known to $1.5 \%$ each in the central region and to $3 \%$ in the forward-backward region from the measurement of the position of the $\rho$ invariant mass peak. This systematic error is common to $\tau^{-} \rightarrow \rho^{-} \nu_{\tau}$ and $\tau^{-} \rightarrow \pi^{-}\left(\mathrm{K}^{-}\right) \nu_{\tau}$ and changes the polarization in the same direction.

The uncertainty in the resolution of the tracking chamber results in an uncertainty on the correction for the effects of charge confusion on the $\mathcal{P}_{\tau}(\cos \theta)$ distribution. It is conservatively estimated to be half the size of the correction itself. This systematic is common to all channels except $\tau^{-} \rightarrow \mu^{-} \bar{\nu}_{\mu} \nu_{\tau}$ for which the charge is defined unambiguously.

There are two theoretical errors relevant to the polarization measured using hadronic $\tau$ decays. The first one is related to the uncertainty in the matrix clement of the $\tau^{-} \rightarrow \mathrm{a}_{1}^{-} \nu_{\tau}$ decay [3]. It affects the $\tau^{-} \rightarrow \mathrm{a}_{1}^{-} \nu_{\tau}$ results and to a much lesser extent the $\tau^{-} \rightarrow \rho^{-} \nu_{\tau}$ results (via uncertainty in the shape of the dominant background). The second error comes from the uncertainty due to structure dependent radiation [24] and affects mostly the $\tau^{-} \rightarrow \pi^{-}\left(\mathrm{K}^{-}\right) \nu_{\tau}$ channel. The uncertainty in the rate of $\tau^{-} \rightarrow \pi^{-}\left(\mathrm{K}^{-}\right) \nu_{\tau} \gamma$ events also increases the background for $\tau^{-} \rightarrow \rho^{-} \nu_{\tau}$.

The systematic errors on $\mathcal{P}_{T}$ depend on the production polar angle and are therefore estimated independently for each $\cos \theta$ bin. Table 1 summarizes the study of the systematic errors for a particular $\cos \theta$ interval.

\subsection{Measurement of polarization using the acolinearity}

The acolinearity between the decay products of two the $\tau$ leptons produced in $\mathrm{Z} \rightarrow \tau^{+} \tau^{-}(\gamma)$ decays is also used to measure $\mathcal{P}_{\tau}$ [9]. We use only the 19911992 data sample for this measurement.

The data sample selected for this analysis consists of $\tau^{-} \rightarrow \pi^{-}\left(\mathrm{K}^{-}\right) \nu_{\tau}$ decays recoiling against one-prong $\tau$ decays. The acolinearity, $\varepsilon$, is defined as $\varepsilon=\pi-$ 


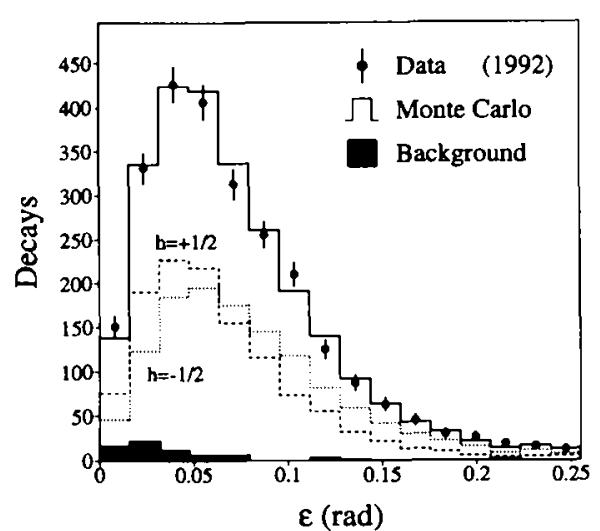

Fig. 5. Spectrum for $\tau^{-} \rightarrow \pi^{-} \nu_{\tau}$ decays as a function of $\varepsilon$ for 1992 data sample. Also shown are the Monte Carlo best fit, the contribution from each helicity, and non- $\tau$ background.

$\alpha_{12}$, where $\alpha_{12}$ is the angle between the $\pi^{-}$track and the charged track in the opposite event hemisphere. The fiducial volume is restricted to the region of polar angle $|\cos \theta|<0.72$. The acolinearity spectrum is shown in Fig. 5, together with the best fit Monte Carlo distributions.

The angular resolution is limited by the uncertainty in the position of the $\mathrm{e}^{+} \mathrm{e}^{-}$interaction point along the beam axis. This uncertainty arises mostly from the finite bunch length, which was measured with $\mathrm{Z} \rightarrow$ $\mathrm{e}^{+} \mathrm{e}^{-}(\gamma)$ events to be $10.1 \pm 0.1 \mathrm{~mm}$ and $7.40 \pm 0.06$ $\mathrm{mm}$ in 1991 and 1992, respectively. The uncertainties in these numbers as well as the uncertainty in the position of the center of interaction region are the main source of the systematic error for the acolinearity method. Uncertainties due to the selection procedure, the background and structure dependent final state radiation are similar to the single $\tau$ method and are listed in Table 1.

The results of the polarization measurement using the acolinearity are combined with the other polarization results. Due to the fact that the same events can enter both the acolinearity measurement and the measurement using the $\tau^{-} \rightarrow \pi^{-}\left(\mathrm{K}^{-}\right) \nu_{\tau}$ energy spectrum, the two results are statistically correlated. This correlation is accounted for in the combined result.

\subsection{Fitting procedure for $\mathcal{A}_{\tau}$ and $\mathcal{A}_{\mathrm{e}}$}

In order to fit $\mathcal{A}_{r}$ and $\mathcal{A}_{e}$ using Eq. (2) the corrections due to initial and final state radiation, $\gamma$-exchange and $\gamma$ Z-interference must be first applied to the data. These corrections are calculated for each $\cos \theta$ bin using the ZFITTER program [25]. The calculations are done for every $\sqrt{s}$ point and averaged weighted by the integrated luminosity at these points. The corrected $\mathcal{P}_{\tau}(\cos \theta)$ dependence is then used to determine $\mathcal{A}_{\tau}$ and $\mathcal{A}_{e}$ for the individual $\tau$ decay channels and the acolinearity method. The results are presented in Table 2 and show consistency of the measurements among the various decay channels. The measurements of $\mathcal{P}_{\tau}(\cos \theta)$ for each $\tau$ decay channel and acolinearity method are then combined bin-by-bin in $\cos \theta$. In the combination we account for the statistical correlation between acolinearity method and single $\tau$ method as well as for the statistical correlation in the single $\tau$ method when both $\tau$ decays in an event are selected for the polarization measurement. The systematic error is obtained by the propagation of the individual uncertainties to the final result. This procedure accounts for the systematics common to the individual channels. The measured values of $\mathcal{P}_{\tau}$ together with the corresponding corrections, statistical and systematic errors are presented in Table 3. $\mathcal{A}_{r}$ and $\mathcal{A}_{e}$ as determined by fitting Eq. (2) to the corrected $\mathcal{P}_{\tau}$ values are

$$
\begin{aligned}
& \mathcal{A}_{\tau}=0.150 \pm 0.013 \pm 0.009 \\
& \mathcal{A}_{\mathrm{e}}=0.157 \pm 0.020 \pm 0.005
\end{aligned}
$$

The first error is statistical and the second is systematic. The statistical error includes both data and Monte Carlo statistics. The correlation coefficient between $\mathcal{A}_{r}$ and $\mathcal{A}_{e}$ in the fit is +0.036 . Fitting Eq. (2) to the corrected data with the assumption $\mathcal{A}_{\boldsymbol{r}}=\mathcal{A}_{\mathrm{e}}$ we obtain

$\mathcal{A}_{e-\tau}=0.152 \pm 0.011 \pm 0.007$.

The corrected $\mathcal{P}_{\tau}$ points together with the best fit curves are shown in Fig. 6.

In the calculation of the systematic errors we account for bin-to-bin correlations separately for each uncertainty source. The charge confusion errors as well as the errors from theory are assumed to be fully correlated; the calibration errors are correlated only for pairs of $\cos \theta$ bins of opposite sign (and there is no correlation between neighboring bins); the background errors are assumed to be uncorrelated. For the selection errors we can not reliably estimate the correlation, therefore we make the worst-case assumption that they are fully correlated when calculating $\Delta \mathcal{A}_{\text {r }}$ 
Table 2

$\mathcal{A}_{T}$ and $\mathcal{A}_{\mathrm{e}}$ for each channel measured during the 1990-1993 running periods. The errors correspond to data and Monte Carlo statistics. The quoted errors do not take into account statistical correlations arising when both event hemispheres are used for the fit.

\begin{tabular}{lllllll}
\hline & $\mathrm{e}^{-\bar{\nu}_{\mathrm{e}} \nu_{\tau}}$ & $\mu^{-} \bar{\nu}_{\mu} \nu_{\tau}$ & $\pi^{-}\left(\mathrm{K}^{-}\right) \nu_{\tau}$ & $\rho^{-} \nu_{\tau}$ & $\mathrm{a}_{\mathbf{1}}^{-} \nu_{\tau}$ & acolinearity \\
\hline $\mathrm{N}_{\text {decays }}$ & 13686 & 14343 & 11109 & 21760 & 2905 & 3774 \\
$\mathcal{A}_{\tau} \times 10^{2}$ & $11.4 \pm 4.9$ & $17.0 \pm 4.5$ & $14.3 \pm 2.2$ & $15.5 \pm 1.7$ & $25.4 \pm 12.8$ & $11.4 \pm 6.5$ \\
$\mathcal{A}_{\mathrm{e}} \times 10^{2}$ & $25.3 \pm 7.4$ & $22.2 \pm 6.4$ & $12.8 \pm 3.5$ & $15.1 \pm 2.7$ & $24.0 \pm 21.1$ & $13.5 \pm 9.8$ \\
\hline
\end{tabular}

Table 3

The combined dependence of $\mathcal{P}_{\tau}$ on polar angle. $\delta \mathcal{P}_{\tau}^{\mathrm{QED}}$ represents a correction to $\mathcal{P}_{\tau}$ due to initial and final state radiation, $\gamma$-exchange and $\gamma Z$-interference. The correction is to be added to the measured values of $\mathcal{P}_{\tau} . \mathcal{P}_{\tau}, \delta \mathcal{P}_{\tau}^{\mathrm{QED}}$ and corresponding statistical and systematic errors for the events in which charge is not defined are shown in the last row. The theoretical error on $\mathcal{P}_{\tau}$ is found to be 0.0007 for all $\cos \theta$ bins.

\begin{tabular}{|c|c|c|c|c|c|c|c|}
\hline $\cos \theta$ range & $\mathcal{P}_{\boldsymbol{\tau}}$ & $\delta \mathcal{P}_{\tau}^{\mathrm{QED}}$ & $\Delta \mathcal{P}_{\tau}^{\text {stat }}$ & $\Delta \mathcal{P}_{\tau}^{\text {sel }}$ & $\Delta \mathcal{P}_{\tau}^{\mathrm{bg}}$ & $\Delta \mathcal{P}_{\tau}^{\text {cal }}$ & $\Delta \mathcal{P}_{\tau}^{\text {chrg }}$ \\
\hline$[-0.92,-0.72]$ & -0.005 & +0.0006 & 0.061 & 0.0098 & 0.0140 & 0.0250 & 0.0064 \\
\hline$[-0.72,-0.55]$ & -0.048 & +0.0000 & 0.040 & 0.0045 & 0.0042 & 0.0126 & 0.0021 \\
\hline$[-0.55,-0.35]$ & -0.002 & -0.0010 & 0.038 & 0.0045 & 0.0042 & 0.0128 & 0.0021 \\
\hline$[-0.35,-0.12]$ & -0.082 & -0.0027 & 0.039 & 0.0046 & 0.0043 & 0.0129 & 0.0015 \\
\hline$[-0.12,+0.12]$ & -0.112 & -0.0045 & 0.036 & 0.0048 & 0.0038 & 0.0137 & 0.0000 \\
\hline$[+0.12,+0.35]$ & -0.201 & -0.0054 & 0.039 & 0.0046 & 0.0040 & 0.0127 & 0.0015 \\
\hline$[+0.35,+0.55]$ & -0.308 & -0.0054 & 0.037 & 0.0045 & 0.0042 & 0.0128 & 0.0021 \\
\hline$|+0.55,+0.72|$ & -0.275 & -0.0053 & 0.039 & 0.0045 & 0.0045 & 0.0130 & 0.0021 \\
\hline$i+0.72,+0.92 i$ & -0.265 & -0.0055 & 0.057 & 0.0098 & 0.0250 & 0.0250 & 0.0064 \\
\hline no charge & -0.143 & -0.0045 & 0.032 & 0.0048 & 0.0046 & 0.0100 & 0.0000 \\
\hline
\end{tabular}

Table 4

Summary of the statistical and systematic errors on $\mathcal{A}_{e}, \mathcal{A}_{\tau}$ and $\mathcal{A}_{e-\tau}$.

\begin{tabular}{llllllll}
\hline & $\begin{array}{l}\text { DATA } \\
\text { statistics }\end{array}$ & $\begin{array}{l}\text { Monte Carlo } \\
\text { statistics }\end{array}$ & selection & background & calibration & $\begin{array}{l}\text { charge } \\
\text { confusion }\end{array}$ \\
\hline $\mathcal{A}_{\tau}$ & 0.012 & 0.004 & 0.005 & 0.002 & 0.007 & 0.000 & 0.001 \\
$\mathcal{A}_{\mathbf{e}}$ & 0.019 & 0.007 & 0.002 & 0.002 & 0.000 & 0.004 & 0.000 \\
$\mathcal{A}_{\mathbf{e}-\tau}$ & 0.010 & 0.004 & 0.004 & 0.001 & 0.005 & 0.001 & 0.001 \\
\hline
\end{tabular}

and uncorrelated when calculating $\Delta \mathcal{A}_{e}$. The breakdown of the errors on $\mathcal{A}_{\tau}$ and $\mathcal{A}_{e}$ is given in Table 4.

\section{Conclusions}

From the measurement of $\mathcal{A}_{r}$ and $\mathcal{A}_{\mathrm{e}}$ we derive the ratio of vector to axial-vector weak neutral couplings for electrons and taus to be

$$
\begin{aligned}
& g_{\mathrm{Ve}_{e}} / g_{\mathrm{Ae}}=0.0791 \pm 0.0099 \pm 0.0025 \\
& g_{\mathrm{V}_{\tau}} / g_{\mathrm{A} \tau}=0.0752 \pm 0.0063 \pm 0.0045
\end{aligned}
$$

The first error includes data and Monte Carlo statistics, the second one is a combination of all systematic uncertainties. This measurement supports the hypothesis of $e-\tau$ universality of the weak neutral current. It agrees with other measurements of $\mathcal{P}_{\tau}$ performed at LEP [26-28] and has reduced errors.

Assuming lepton universality we derive the ratio of vector to axial-vector weak neutral couplings for leptons and the effective electroweak mixing angle to be

$$
\begin{aligned}
& g_{\mathrm{V}} / g_{\mathrm{A}}=0.0763 \pm 0.0054 \pm 0.0033 \\
& \sin ^{2} \theta_{w}^{\text {eff }}=0.2309 \pm 0.0016
\end{aligned}
$$




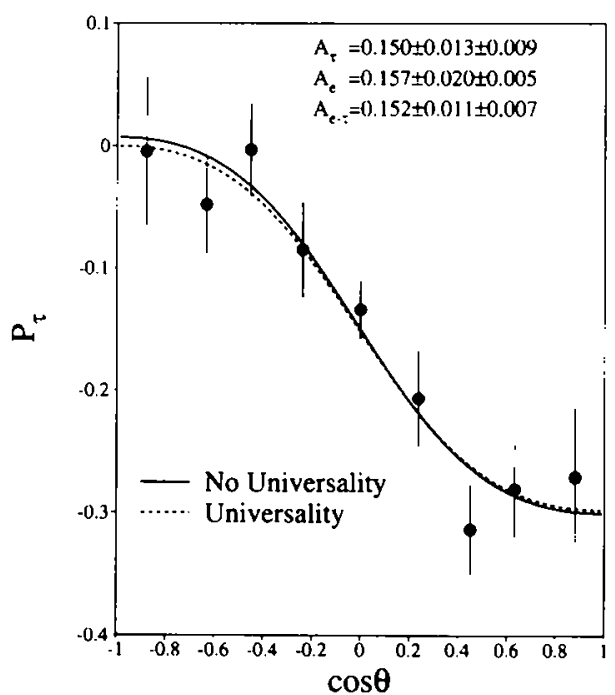

Fig. 6. The measured dependence $\mathcal{P}_{\tau^{-}}(\cos \theta)$ for all channels combined (dots). The errors include data and Monte Carlo statistics only. $\mathcal{P}_{\tau^{-}}(\cos \theta)$ is corrected bin-by-bin for initial and final state radiation, $\gamma$-exchange and $\gamma \mathrm{Z}$-interference. The solid and dashed lines represent the fits using Eq. (2) with and without assumption of lepton universality.

This is consistent with other L3 measurements of $\sin ^{2} \theta_{\mathrm{w}}^{\text {eff }}$ extracted from the study of the $\mathrm{Z}$ lineshape and forward-backward charge asymmetries in the processes $\mathrm{Z} \rightarrow \mathrm{e}^{+} \mathrm{e}^{-}(\gamma), \mathrm{Z} \rightarrow \mu^{+} \mu^{-}(\gamma), \mathrm{Z} \rightarrow$ $\tau^{+} \tau^{-}(\gamma)$ and $Z \rightarrow b \bar{b}[29,30]$.

\section{Acknowledgement}

We wish to express our gratitude to the CERN accelerator divisions for the excellent performance of the LEP machine. We acknowledge the efforts of all engineers and technicians who have participated in the construction and maintenance of this experiment.

\section{References}

[1] S. Jadach, Z. Was et al., in: Z Physics at LEPI, CERN Report CERN-89-08, Vol. 1, eds. G. Altarelli, R. Kleiss and C. Verzegnassi (CERN, Geneva, 1989) p. 235.

[2] S.L. Glashow, Nucl. Phys. 22 (1961) 579; S. Weinberg, Phys. Rev. Lett. 19 (1967) 1264; A. Salam, Elementary Particle Theory, Ed. N. Svartholm, (Almquist and Wiksell, Stockholm, 1968) p. 367.

[3] ARGUS Collaboration, H. Albrecht et al., Phys. Lett. B 250 (1990) 164;

H. Albrecht et al., Z. Phys. C 58 (1993) 61.
[4] ARGUS Collaboration, H. Albrecht et al., Phys. Lett. B 246 (1990) 278;

H. Albrecht et al., Phys. Lett. B 316 (1993) 608;

H. Albrecht et al., Preprint DESY 94-100 (June 1994).

[5] ALEPH Collaboration, D. Buskulic et al., Phys. Lett. B 321 (1994) 168.

[6] Y.S. Tsai, Phys. Rev. D 4 (1971) 2821.

17] K. Hagiwara, A.D. Martin and D. Zeppenfeld, Phys. Lett. B 235 (1990) 198.

[8] A. Rougé, Z. Phys. C 48 (1990) 75.

19] R. Alemany et al., Nucl. Phys. B379 (1992) 3

[10] L3 Collaboration, O. Adriani et al., Phys. Lett. B 294 (1992) 466.

[11] L3 Collaboration, B. Adeva et al., Nucl. Instr. Meth. A 289 (1990) 35;

O. Adriani et al., Nucl. Instr. and Meth. A 302 (1991) 53;

K. Deiters et al., Nucl. Instr. and Meth. A 323 (1992) 162;

J.A. Bakken et al., Nucl. Instr. and Meth. A 275 (1989) 81.

[12] S. Jadach, B.F.L. Ward and Z. Was, Comput. Phys. Commun. 66 (1991) 276;

R. Kleiss et al., in: Z Physics at LEP1, Vol. 3, CERN Report CERN 89-08 (1989) p. 1.

[13] R. Brun et al., GEANT 3, CERN DD/EE/84-1 (Revised), September 1987.

[14] H. Fesefeldt, RWTH Aachen Preprint PITHA 85/02 (1985).

[15] T. Sjöstrand and M. Bengtsson, Comput. Phys. Commun. 43 (1987) 367;

T. Sjöstrand et al., in: Z Physics at LEP1, Vol. 3, CERN Report CERN 89-08 (1989), p. 143.

[ 16$]$ P. Privitera, Phys. Lett. B 308 (1993) 163.

[17] M. Davier et al., Phys. Lett. B 306 (1993) 411.

[18] J.H. Kühn and E. Mirkes, Phys. Lett. B 286 (1992) 381. J.H. Kühn and E. Mirkes, Z. Phys. C 56 (1992) 661.

[19] N. Isgur et al., Phys. Rev. D 39 (1989) 1357.

[20] M. Feindt, Z. Phys. C 48 (1990) 681.

[21] L3 Collaboration, B. Adeva et al., Phys. Lett. B 265 (1991) 451.

[22] L3 Collaboration, M. Acciarri et al., A Measurement of exclusive Branching Fractions of Hadronic One-Prong Tau Decays, in preparation.

[23] Particle Data Group, K. Hikasa et al., Review of Particle Properties, Phys. Rev. D 45 (1992) 1.

[24] M. Finkemeier, Ph.D. Thesis, University of Karlsruhe (1994).

[25] D. Bardin et al., FORTRAN package ZFITTER; CERN-TH 6443/92; Z. Phys. C 44 (1989) 493; Nucl. Phys. B 351 (1991) 1; Phys. Lett. B 255 (1991) 290.

[26] ALEPH Collaboration, D. Buskulic et al., Z. Phys. C 59 (1993) 369.

[27] DELPHI Collaboration, P. Abreu et al., Z. Phys. C 55 (1992) 555.

[28] OPAL Collaboration, R. Akers et al., Preprint CERN-PPE 94-120, July 1994;

G. Alexander et al., Phys. Lett. B 266 (1991) 201

[29] L3 Collaboration, M. Acciarri et al., Z. Phys. C 62 (1994) 551.

[30] L3 Collaboration, M. Acciarri et al., Preprint CERN-PPE 94-89, June 1994 